\title{
DYNAMIC PRICING POLICY USING PRESERVATION TECHNOLOGY WITH STOCK AND PRICE SENSITIVE DEMAND
}

\author{
Uttam Kumar Khedlekar ${ }^{1}$, Priyanka Singh ${ }^{2}$, Neelesh Gupta ${ }^{3}$, \\ ${ }^{1}$ Department of Mathematics and Statistics \\ Dr. Hari Singh Gour Vishwavidyalaya, Sagar M.P. India \\ (A Central University) \\ e-mail: uvkkcm@yahoo.co.in, \\ e-mail: priyanka1singh2013@gmail.com, \\ e-mail: neeleshgupta12414@gmail.com
}

\begin{abstract}
This paper aims to develop a dynamic pricing policy for deteriorating items with price and stock dependent demand. In declining market demand of items decreases with respect to time and also after a duration items get outdated. In this situation it needs a pricing policy to sale the items before end season. The proposed dynamic pricing policy is applicable for a limited period to clease the stock. Policy decision regarding the selling price could aggressively attracts the costumers. Objectives are to maximize the profit/revenue, pricing strategy and economic order level for such a stock dependent and price sensitive items. We are giving numerical example and simulation to illustrate the proposed model.
\end{abstract}

Key words and Phrases: Inventory, stock and price sensitive dependent demand, preservation technology, optimal profit.

\section{INTRODUCTION}

Inventory management that maintains an adequate balance between demand and supply is an essential need for any business. The nature of items plays an important role in inventory management. The items, for this purpose, can be affected by and at the same time can affect several logistical and market forces like production, demand, transportation, price, etc. Further, the life of items and the nature of deterioration of item characteristics also play a very important role. Some inventory items are perishable over long duration of time, while some perish over small time interval. In addition, inventory problem is also affected by the inflation rate, shortage of items, order size, price fluctuations, discount strategies

2020 Mathematics Subject Classification: 90B05, 90B30, $90 \mathrm{~B} 50$.

Received: 10-11-2018, accepted: 12-06-2020. 
and marketing plans. In the present e-shopping environment, customers have many options to compare the similar products on the basis of merits, price, and according to place of order. In this process one can save expenditure on shopping and time both. The price reduction with e-shopping is a tool to generate the excess demand of item. In super market, presence of larger quantity of item attracts the customer to buy more items; this phenomenon reveals that stock dependent demand also can be a tool to generate the demand. Pricing strategy is a tool that attracts costumer to buy more. Within (1955) was first to integrate the concept of inventory theory to the concept of price theory and simultaneously determined the price prospect and order quantity of a retailer. In a situation when all other assumptions of the EOQ model are valid, he took demand as a price sensitive parameter. You (2005) picked up the problem of joint determination of order size and optimal price for the perishable inventory system subjected to condition that when demand is exponentially decreasing and price sensitive. It is with the assumptions that a decision maker gets an optimal level to adjust price before entering into next time interval to influence demand and revenue both. Taleizadeh et al. (2015) revisited the idea of replenishment frequency after each replenishment cycle and production rate in vendor managed inventory system with deterioration. Internal process of dengue virus transmission in the human body studied by Nuraini et al. (2007). They developed a mathematical model an analyzed the dynamic of dengue virus and supported by a numerical example.

Son and Ikuta (2007) dealt with a problem of selecting profitable orders to accept out of sequentially arriving customers in a custom production company where only one customer is allowed to be held. The delay cost would be paid for every order not completed up to its due date. Inventory cost plays a vital role in inventory management. Sarkar and Sarkar (2013a) expanded an inventory model for deteriorating items with stock-dependent demand. This is applicable in which time varying backlogging rate and time varying deterioration rate. They determined the optimal cycle length of each product such that the expected total cost including holding, shortage, ordering, deterioration and opportunity cost. Further, they provided the necessary and sufficient conditions for existence and uniqueness of the optimal solution some of the literature are giving in references like Sarkar and Sarkar $(2013 b, c)$, Sarkar and Goswami(2012), Tjandra et al. (2015), Sarkar et al. (2011), Masjkur, et al. (2018) Taleizadeh et al.(2015), Khedlekar and Shukla (2013).

Expenditure sources like ordering cost, safety, lead time and numbers of lots are the integral parts of decision making. An integrated inventory model focusing on these issues has been discussed by Lo (2007). Skouri and Papachristos (2002) also attempted the temporary price reductions to resellers when manufacturers, distributors, wholesalers are offer trade promotion. Khedlekar et al. (2013) computed the time periods before and after disrupt a production system. Further useful contributions on pricing theory, are due to Chen and Chen (2007).

The EOQ model designed by Hou and Lin (2006) reflects how a demand pattern which is price and stock dependent affects the discount in cash. You (2007) 
investigated a perishable inventory problem wherein a firm purchases and prices a service or a perishable item before a sales season and sells it through a reservation system during the sales season. Taking the cancellation phenomenon and the effect of waiting time on demand into account, he developed two continuous time models to respectively solve a problem with a time independent refund. A thought from the literature survey appears rarely considered stock dependent with price sensitive demand in research contributions. In view of above literature, we have motivated to design a dynamic model by considering price sensitive and stock dependent demand. We assumed the market is in decline nature and thats why we are reducing the selling price and generate the excess demand to increase the revenue. We have divided the sale session into $n$ equal parts and find out the replenishment size and number of price settings.

\section{Assumptions and notations}

Suppose in an inventory system where a company purchases the items before starting of a sales season and sales it to the customers in time horizon $L$ which is divided into $n$ equal parts i.e. $T=L / n$. The inventory managers decides to change the selling price $n$ times in $n$ equal intervals $[0, T],[T, 2 T] \cdots[(j-1) T, j T]$. $\cdots[(n-1) T, n T]$ and the respective prices are $p_{1}, p_{2}, \cdots p_{j} \cdots p_{n}$ in decreasing order. Suppose $\alpha>0$ is the intersection of the demand curve (initial demand) of the item and if $\eta$ is parameter of contribution of stock in demand which is proportional to it and $\beta$ is parameter contribution of price in demand. If at time $t$ on hand inventory is $I_{j}(t)$ and the demand of the item is $d_{j}(p, t)=\alpha-\beta p_{j}+\eta I_{j}(t)$.

If sold quantity of items are $Q(j)$ for period the index $j$. If there is some cost occurs to change the selling price once like advertisers and displaying the change in price in super market say which is $A$, and $c$ is unit purchasing cost. The holding cost of inventory is $h$ unit per unit time. It is assumed that demand increases accordingly reduction in selling price and proposition to on hand inventory $I_{j}(t)$. The following notations are used to formulate the proposed model.

- $\lambda(\alpha)$ preservation technology function $\left(\lambda(\alpha)=\lambda_{0} e^{-\delta \alpha}\right)$

- $L$ sale season,

- $Q$ order quantity,

- $\eta$ parameter of stock dependent demand $\eta>0$,

- $n$ number of permissible price settings,

- $T$ length of period for one price setting, $T=L / n$,

- $j$ period $\mathrm{j}$ refers the time interval $[(j-1) T, j T]$,

- $c$ unit purchasing cost,

- A price setting cost per price change,

- $p_{j} j^{t h}$ selling price of product,

- $d_{j}(p, t)$ demand of product in $j^{t h}$ interval $[(j-i) t, j t]$. 


\section{The Mathematical Model}

The rate of decay in inventory due to demand $\left(\alpha-\beta p_{j}+\eta I_{j}(t)+\lambda(\alpha) I_{j}(t)\right)$ in the interval $[0, T]$, satisfied the following differential equation.

$$
\frac{d}{d t} I_{j}(t)=-\left(\alpha-\beta p_{j}+\eta I_{j}(t)+\lambda(\alpha) I_{j}(t)\right)
$$

where $0 \leq t \leq T$ and boundary condition $I_{j}(0)=I_{j-1}(T)$

On hand inventory denoted by $I_{j}(t)$ at time $t$ expressed as following equation

$$
I_{j}(t)=-q_{j} e^{(\eta+\lambda(\alpha)) t}-\frac{\alpha-\beta p_{j}}{\eta}\left(1-e^{(\eta+\lambda(\alpha)) t}\right)
$$

Sold items $q_{j}(t)$ at $j^{\text {th }}$ period is

$$
q_{j}(t)=-x^{-1} q_{j-1}-\frac{\alpha-\beta p_{j}-1}{\eta+\lambda(\alpha)}\left(1-x^{-1}\right), \text { where } x=e^{-(\eta+\lambda(\alpha)) T}
$$

If $q_{1}=Q$, then above equation leads to

$$
q_{j}(t)=x^{-(j-1)} Q-\frac{\alpha}{\eta+\lambda(\alpha)} 1-x^{-(j-1)}+\beta\left(1-x^{-1}\right) \sum_{i=2}^{j}\left(x^{-(j-1)} p_{(i-1)}\right.
$$

Sale quantity of stock in interval $[(j-1) T, j T]$ is just difference of $q_{j}$ and $q_{j+1}$, where $j=1,2,3, \ldots \ldots n$

$$
\begin{gathered}
\delta q_{j}=q_{j}-q_{j+1} \\
\delta q_{j}=x^{j}(x-1)(\alpha+(\eta+\lambda(\alpha)) Q)-\frac{\beta\left(1-x^{-1}\right) p_{j}}{\eta+\lambda(\alpha)} \\
+\frac{\beta}{\eta+\lambda(\alpha)}\left(1-x^{-1)^{2}}\left(\sum_{i=1}^{j-1} x^{-(j-i-1)}\left(1-x^{-1}\right) p_{i-1}\right)\right. \\
I_{j}(t)=e^{(\eta+\lambda(\alpha)) t}\left(x^{-(j-1)} Q-\frac{\alpha\left(1-x^{-(j-1)}\right)}{(\eta+\lambda(\alpha))}+\frac{\left(1-x^{-1}\right) \beta\left(\sum_{i=2}^{j} x^{-(j-1)} p_{(i-1)}\right)}{\eta}\right) \\
-\frac{\left(\alpha-\beta p_{j}\right)}{\eta}\left(1-e^{(\eta+\lambda(\alpha)) t}\right)
\end{gathered}
$$

Boundary condition $I_{j-1}(t)=0$, leads to

$$
Q(n)=\frac{1}{(\eta+\lambda(\alpha))}\left(\alpha\left(x^{n}-1\right)-\beta\left(1-x^{-1}\right) \sum_{i=1}^{n} p_{i}+\beta(1-x-1) \sum_{i=2}^{n} x^{-(i-1)} p_{i-1}\right)
$$

Revenue $R(n)$ when price changes $n$ times over time $L$ is

$$
R(n)=(x-1)\left(\frac{\alpha}{(\eta+\lambda(\alpha))}\right) \sum_{j=1}^{n} p_{j}+\frac{\beta\left(1-x^{-1}\right)^{2}}{(\eta+\lambda(\alpha))}\left(\sum_{j=2}^{n} \sum_{i=1}^{j-1} p_{i} p_{j}\right)-\frac{\beta}{\eta+\lambda(\alpha)} \sum_{i=1}^{n} p_{i}^{2}
$$


Holding cost $H(n)$ over time $L$ is

$$
H(n)=\sum_{j=1}^{n} \int_{0}^{T} h I_{j}(t) d t
$$

Profit function $F(n, \mathbf{p})$ is the difference of Revenue and total incurred cost

$$
F(n, \mathbf{p})=R(n)-H(n)-c Q(n)-n A
$$

\section{Special Cases}

If $\mathbf{n}=\mathbf{1}$

If firm is not permit to change the selling price in entire season. It means the business is running with only one price in sale season $[0, L]$. That is $n=1$ and $\mathbf{p}=\left(p_{1}\right)$. on putting $n=1$ in equation (3.7) leads to

$$
\begin{gathered}
\frac{\partial}{\partial p_{1}} F(1, \mathbf{p})=-\frac{2 \beta}{\eta+\lambda(\alpha)} p_{1}+\frac{x-1}{(\eta+\lambda(\alpha))^{2}}((\alpha+c \beta)(\eta+\lambda(\alpha))+h \beta) \\
-\frac{h T \beta}{\eta+\lambda(\alpha)}=0 \\
\frac{\partial^{2}}{\partial p_{1}^{2}} F(1, \mathbf{p})=\frac{-2 \beta}{\eta} \leq 0, \quad p_{1}=\frac{\alpha(\eta+\lambda(\alpha))+c \beta \eta+h}{(\eta+\lambda(\alpha))}+\frac{h T}{2(1-x)}
\end{gathered}
$$

and equation (3.4) leads to

$$
Q(1)=\frac{\beta T h}{2(\eta)}+\frac{(x-1)(\alpha \eta-c(\eta+\lambda(\alpha)) \beta-h)}{2(\eta+\lambda(\alpha))^{2}}
$$

If $\mathbf{n}=\mathbf{2}$

If firm decide to change the selling price once over the entire season $L$. It means the business is running with two prices $p_{1}$ and $p_{2}$ in intervals $[0, T]$ and in $[T, 2 T]$, where $T=L / 2$. That is $n=2$ and $\mathbf{p}=\left(p_{1}, p_{2}\right)$, thus on putting $n=2$ the equation (3.7) leads to

$$
\frac{\partial}{\partial p_{1}} F(2, \mathbf{p})=0, \frac{\partial}{\partial p_{2}} F(2, \mathbf{p})=0
$$

Above two equations evaluates two prices $p_{1}$ and $p_{2}$ in decereasing order. To obtain optimization conditions we have find followings

$$
\begin{aligned}
& A_{11}=\frac{\partial^{2}}{\partial p_{1}^{2}} F(2, \mathbf{p}), A_{12}=\frac{\partial^{2}}{\partial p_{1} \partial p_{2}} F(2, \mathbf{p}) \\
& A_{21}=\frac{\partial^{2}}{\partial p_{2} \partial p_{1}} F(2, \mathbf{p}), A_{22}=\frac{\partial^{2}}{\partial p_{2}^{2}} F(2, \mathbf{p})
\end{aligned}
$$


and Hessian matrix $H_{22}$ is

and quantity $Q(2)$ is

$$
H_{22}=\left(\begin{array}{ll}
\frac{\partial^{2} F(2, \mathbf{p})}{\partial p_{1} \partial p_{1}}, & \frac{\partial^{2} F(2, \mathbf{p})}{\partial p_{1} \partial p_{2}} \\
\frac{\partial^{2} F(2, \mathbf{p})}{\partial p_{1} \partial p_{2}}, & \frac{\partial^{2} F(2, \mathbf{p})}{\partial p_{2} \partial p_{2}}
\end{array}\right) .
$$

$$
Q(2)=\frac{\beta T h}{2(\eta+\lambda(\alpha))}+\frac{2(x-1)(\beta h+c \beta(\eta+\lambda(\alpha))-\alpha \eta)}{2(\eta+\lambda(\alpha))^{2}}+\frac{h T \beta}{(\eta+\lambda(\alpha))}
$$

If $\mathbf{n}=\mathbf{3}$

If firm decide to change the price twice that is in this case $n=3$, and three prices $p_{1}, p_{2}, p_{3}$ are applied in the intervals $[0, T],[T, 2 T]$ and $[2 T, 3 T]$ respectively, where $T=L / 3$ and $\mathbf{p}=\left(p_{1}, p_{2}, p_{3}\right)$. Equation (3.7) leads for $n=3$,

$$
\frac{\partial}{\partial p_{1}} F(3, \mathbf{p})=0, \frac{\partial}{\partial p_{2}} F(3, \mathbf{p})=0, \frac{\partial}{\partial p_{3}} F(3, \mathbf{p})=0
$$

Above three equations evaluates three prices $p_{1}, p_{2}$ and $p_{3}$. To obtain optimization conditions we have to find followings.

$$
\begin{aligned}
& A_{11}=\frac{\partial^{3}}{\partial p_{1}^{3}} F(3, \mathbf{p}), A_{12}=\frac{\partial^{3}}{\partial p_{1} \partial p_{2}^{2}} F(3, \mathbf{p}), A_{13}=\frac{\partial^{3}}{\partial p_{1} \partial p_{3}^{2}} F(3, \mathbf{p}), \\
& A_{21}=\frac{\partial^{3}}{\partial p_{1}^{2} \partial p_{2}} F(3, \mathbf{p}), A_{22}=\frac{\partial^{3}}{\partial p_{2}^{3}} F(3, \mathbf{p}), A_{23}=\frac{\partial^{3}}{\partial p_{2} \partial p_{3}^{2}} F(3, \mathbf{p}), \\
& A_{31}=\frac{\partial^{3}}{\partial p_{1}^{3}} F(3, \mathbf{p}), A_{32}=\frac{\partial^{3}}{\partial p_{1} \partial p_{1} \partial p_{2}} F(3, \mathbf{p}), A_{33}=\frac{\partial^{3}}{\partial p_{3}^{3}} F(3, \mathbf{p}) .
\end{aligned}
$$

and to find rest third derivatives of $F(3, \mathbf{p})$, and $Q(3)$ could be found by equation (3.5) and equation (3.7) . Thus the Hessian matrix $H_{33}$ is,

$$
H_{33}=\left(\begin{array}{lll}
A_{11} & A_{12} & A_{13} \\
A_{21} & A_{22} & A_{23} \\
A_{31} & A_{32} & A_{33}
\end{array}\right) .
$$

\section{Numerical Example}

Suppose the parameters are $\alpha=900, L=90, \eta=0, \beta=0.2, h=0.005$. If charge for one price change is $A=1000$, then on applying model output for $n=1$ the selling price $p_{1}$ is 55.12 in interval $[0,120]$, and order quantity $Q(1)=$ 1698 and respective profit of the system $F_{1}(1, p)=7285$. If firm has to decide that there will be two selling prices in the time intervals $(0,45]$ and $(45,90]$, then selling price $p_{1}=58.43$, and selling price $p_{2}=51.80$, quantity will be $Q(2)=2034$, and respective profit will be $F_{2}(2, p)=7882$. If firm has to decide to take three selling prices $p_{1}=59.72, p_{2}=55.00, p_{3}=50.85$. The order quantity $Q(3)=2113$, respective profit $F_{3}=6688$. In this example we observed that the optimum profit for $n=2$ is maximum than $n=1$ and $n=3$. Hence the optimum value exists at $n=2$. 


\section{Sensitive Analysis}

Table 1: Sensitivity analysis parameter $(\beta)$ in demand

\begin{tabular}{|c|c|c|c|c|c|c|}
\hline \hline$\beta$ & $n$ & $p_{1}$ & $p_{2}$ & $p_{3}$ & $Q$ & $\mathrm{~F}(\mathrm{n}, \mathbf{P})$ \\
\hline 0.2 & 1 & 287.60 & - & - & 2278 & 598511 \\
\hline 0.2 & 2 & 311.00 & 264.22 & - & 3751 & 607160 \\
\hline 0.2 & 3 & 319.00 & 287.82 & 257.50 & 3763 & 607750 \\
\hline 0.4 & 1 & 175.10 & - & - & 2269 & 12190 \\
\hline 0.4 & 2 & 182.25 & 167.96 & - & 2304 & 12991 \\
\hline 0.4 & 3 & 184.66 & 175.32 & 1666.7 & 2311 & 607750 \\
\hline 0.6 & 1 & 137.60 & - & - & 845 & 9497 \\
\hline 0.6 & 2 & 139.34 & 138.87 & - & 859 & 8637 \\
\hline 0.6 & 3 & 139.92 & 137.82 & 136.4 & 861 & 5050 \\
\hline \hline
\end{tabular}

\section{Discussion}

To simulates the model we use the data set as per second date in table 1 , and set the selling price once, twice and thrice simultaneously. For $n=1,2,3$, therefore no change, one change and twice change in selling price over sale season $L$. The selling price $p_{1}, p_{2}$ and $p_{3}$ are in $[0, T],(T, 2 T],(2 T, 3 T]$ optimal quantity is $Q(3)$ and profit is $F_{3}$ are given in table 1 . For $\beta=.02$, the profits for $n=2$ is higher than $n=1$ and $n=3$. Optimal profit and consumed quantity are very sensitive on parameter $\beta$ which are increasing in terms of $\beta$ increasing, higher initial demand is flexible to put higher selling price initially. However profit and quantity are higher at $n=3$ and $p_{1}>p_{2}>p_{3}$ in decreasing order to sell the entire stock. for rest value of $\beta=0.2,0.4$ and 0.6 the profits are increases number of price setting $n$ increases. So it is still continue to investigate to find optimal price settings and respective profit.

Table 2: Sensitiviy analysis for parameter purchasing cost $(c)$

\begin{tabular}{|c|c|c|c|c|c|c|}
\hline \hline$c$ & $n$ & $p_{1}$ & $p_{2}$ & $p_{3}$ & $Q$ & $\mathrm{~F}(\mathrm{n}, \mathbf{P})$ \\
\hline 350 & 1 & 456.30 & - & - & 6533 & 693025 \\
\hline 350 & 2 & 506.30 & 406.23 & - & 7280 & 771298 \\
\hline 350 & 3 & 524.45 & 456.27 & 388.20 & 7439 & 787148 \\
\hline 400 & 1 & 481.27 & - & - & 4996 & 404804 \\
\hline 400 & 2 & 519.53 & 443.01 & - & 5566 & 450149 \\
\hline 400 & 3 & 533.40 & 481.27 & 429.20 & 5688 & 787148 \\
\hline 450 & 1 & 506.27 & - & - & 3458 & 193458 \\
\hline 450 & 2 & 532.75 & 479.79 & - & 3853 & 214659 \\
\hline 450 & 3 & 542.35 & 506.27 & 470.30 & 3938 & 218158 \\
\hline 500 & 1 & 531.27 & - & - & 1921 & 58986 \\
\hline 500 & 2 & 545.97 & 516.57 & - & 2140 & 64829 \\
\hline 500 & 3 & 551.30 & 531.27 & 511.30 & 64971 & 64971 \\
\hline
\end{tabular}

\section{Discussion}

From table 2, we observed that the $p_{1}, p_{2}, p_{3}$ are increases as well as unit purchasing cast $(c)$ increases this is a strength of the proposed model. This characteristic could be explained as follow. First to note that if unit purchasing cost increases the profit 
reduced accordingly, so to coop up this amount of revenue, proposed model set the higher selling prices $p_{1}, p_{2}, p_{3}$ increases accordingly. For $c=350$, the profit is gradually increases for $n=1, n=2$ and $n=3$, and this phenomenon followed for $c=400, c=450$, and $c=500$.

\section{Conclusion}

In this paper we have developed a dynamic pricing policy and effect of parameters analyzed by computing the selling prices and respective output. It is advised to management keep Longer length of sale season $(L)$ that will provides more number of price settings because of proposed model is highly sensitive on this parameter $(L)$. Unit purchasing cost $(c)$ negatively affects the business, because of that model provides higher selling prices and revenues accordingly. If $\beta$ increases means some higher amounts deduces from $\alpha$, and thus demand decreases therefore profit become less, to coop up this amount of losses, model sets higher selling price accordingly to uplift the profit. This is the strength of the proposed model. Sufficient conditions for optimality as concaveness shown in the model and analyzed the model parameters. On substituting $\eta=0$, the model converted for only price sensitive demand, and on putting $\beta=0$, it converted into only stock dependent model. Theoretical and analytical evidence indicates the existence of an optimal number of changes in the value of selling prices for achieving an optimal profit in any business setup.

One can reformulated the model with variable holding cost and as well as variable deterioration. Also One can divide the sale season in unequal length of time intervals and determine the respective selling prices.

\section{REFERENCES}

[1] Chen, J.M. and Chen, L.T., Periodic pricing and replenishment policy for continuously decaying inventory with multivariate demand, Appl. Math. Model., 31 (2007), 1819-1828.

[2] Dubey, R. and Mishra, L.N., Nondifferentiable multiobjective higher-order duality relations for unified type dual models under type-I functions, Adv. Stud. Contemp. Math., 29(3) (2019), 373-382.

[3] Dubey, R., Deepmala and Mishra, V.N., Higher-order symmetric duality in nondifferentiable multiobjective fractional programming problem over cone constraints, Stat. Opt. Inf. Comp., 8 (2020), 187205.

[4] Dubey, R.V. and Mishra, V.N. Second order multiobjective symmetric programming problem and duality relations under $\left(F, G_{f}\right)$-convexity, Global Journal of Engineering Science and Researches 5(8) (2018), 187-199.

[5] Dubey, R., Mishra, L.N. and Cesarano, C. Multiobjective fractional symmetric duality in mathematical programming with $\left(C, G_{f}\right)$-invexity assumptions, Axioms, 8(3) (2019), 97107 . 
[6] Hou, K.L. and Lin, L.C., An EOQ model for deteriorating items with price and stock dependent selling price under inflation and time value of money, Internat. J. Systems Sci., 37(15) (2006), 1131-1139.

[7] Khedlekar, U. K., Shukla, D. and Chandel, R.P.S., Computational study for disrupted production system with time dependent demand, J. Sc. ES Ind. Res., Vol. 73(5) (2013), 294-301.

[8] Khedlekar, U.K. and Shukla, D., Dynamic pricing model with logarithmic demand, Opsearch, 50(1) (2013), 1-13

[9] Masjkur, M. and Folmer, H., "Bayesian estimation of random parameter models of responses with normal and skew-t distributions evidence from monte carlo simulation", J. Indones. Math. Soc. 24(1) (2018), 27-50.

[10] Nuraini, N., Soewono, E. and Sidarto, K.A., "A mathematical model of dengue internal transmission process", J. Indones. Math. Soc. 13(1) (2007), 123-132.

[11] Skouri, K. and Papachristos, S., A continuous review inventory model, with deteriorating items time-varying demand linear replenishment cost partially time-varying backlogging ,Appl. Math. Model., 26 (2002), 603-617.

[12] Sarkar, B. and Sarkar, S., An improved inventory model with partial backlogging, time varying deterioration and stock-dependent demand, Eco. Mod., 30 (2013a), 924-932.

[13] Sarkar, B. And Sarkar, S., Variable deterioration and demand an inventory model, Eco. Mod., 31 (2013b), 548-556.

[14] Sarkar, M. and Sarkar, B., An economic manufacturing quantity model with probabilistic deterioration in a production system, Eco. Mod., 31 (2013c), 245-252.

[15] Sarkar, B. and Goswami, A., A two-warehouse inventory model with increasing demand and time varying deterioration, cientia Iranica, Transaction E: Ind. Enginee, 19 (2012c), 306-310.

[16] Sarkar, B., Sana, S.S. and Choudhari, K.S., An imperfect production process for time varying demand with in ation and time value of money An EMQ model, Exp. Sys. App., 38 (2011), 13543-13548.

[17] Son, J.D. and Ikuta, S. , Customer selection problem with search cost due date sideline profit and no waiting room, Asia-Pac. J. Ope. Res., 24(5) (2007), 647-666.

[18] Taleizadeh, A.A., Kalantari, S.S., and Csardenas-Barrson, L. E. ,Determining optimal price, replenishment lot size and number of shipments for an EPQ model with rework and multiple shipments, J. Ind. Manag. Optim., 11(4) (2015), 1059-1071.

[19] Taleizadeh, A.A., Noori-daryan, M. and Csardenas-Barrson, L.E., Joint optimization of price, replenishment frequency, replenishment cycle and production rate in vendor managed inventory system with deteriorating items, ,Int. J. Pro. Eco., 159(2015), 285-295.

[20] Tjandra S.S., Pudjaprasetya,S.R. and Wiryanto, L.H., Simulation of analytical transient wave due to downward bottom thrust, J. Indones. Math. Soc. 21(2) (2015), 93-104.

[21] Whitin, T.M., Inventory control and price theory, ,Man. Sci., 24(1) (1955), 61-68.

[22] Vandana, R. V., Deepmala, Mishra, L.N. and Mishra, V.N., Duality relations for a class of amultiobjective fractional programming problem involving support functions, Ame. J. Ope. Res., 8 (2018), 294-311.

[23] You, P.S., Optimal pricing for an advance sales system with price and waiting time dependent demands, ,J. Ope. Res. Soc. Japan, 50(2) (2007), 151-161.

[24] You, P.S., Inventory policy for product with price and time-dependent demand, J. Ope. Res., 56(7) (2005), 870-873. 